\title{
Análise do perfil de utilização de medicamentos ansiolíticos em uma unidade da
}

\section{Farmácia da Família no Sudoeste Baiano}

\author{
Profile analysis of use of anxiolytic medications in a unit of the Family Pharmacy in Southwest
}

Bahia

Análisis del perfil de uso de ansiolíticos en una unidad de la Farmacia de Familia en el Sudoeste de

Bahia

Recebido: 17/11/2021 | Revisado: 26/11/2021 | Aceito: 29/11/2021 | Publicado: 10/12/2021

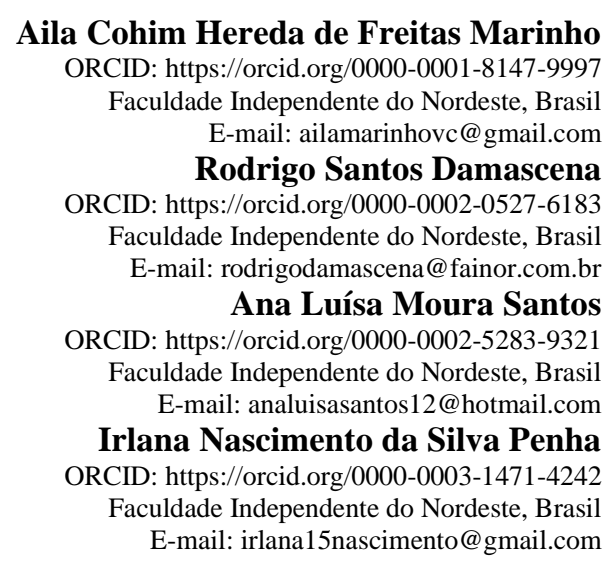

\section{Resumo}

O Uso Racional de Medicamentos deve ser promovido por meio de ações que regulem sua prescrição, dispensação e consumo. Sendo assim, é preocupante o crescente uso de ansiolíticos, principalmente no que diz respeito ao seu uso irracional, o qual pode acarretar tolerância, dependência química e síndrome de abstinência. Esta pesquisa apresenta como objetivo descrever o perfil de utilização dos medicamentos ansiolíticos dispensados em uma Farmácia da Família no Sudoeste Baiano. Trata-se de um estudo descritivo e transversal, com abordagem quantitativa, cujos dados foram extraídos por meio de aplicação de questionários aos usuários desses fármacos, nos meses de agosto e setembro de 2021. A análise dos dados foi realizada através do Statistical Package for the Social Sciences versão 22.0 e apresentada em forma de frequência absoluta e relativa para as variáveis categóricas, além da média e desvio padrão para as variáveis contínuas. Obteve-se como resultado um perfil de uso crônico de benzodiazepínicos, com idade média dos usuários de 53,87 $( \pm 11,10)$ anos, prevalecendo o gênero feminino $(59,34 \%)$, com baixos poder aquisitivo e grau de escolaridade. O medicamento mais utilizado foi o Clonazepam e a patologia com maior indicação, a insônia. Espera-se que os dados obtidos dessa pesquisa possam contribuir com condutas e medidas que propiciem o seu uso racional e auxiliem na efetivação de alternativas de tratamento que visem à melhoria da qualidade de vida desses pacientes, inclusive com a adoção de medidas não farmacológicas.

Palavras-chave: Ansiolíticos; Estudo de utilização de medicamentos; Uso racional.

\begin{abstract}
It is necessary to promote the rational use of medications through actions that regulate their prescription, dispensing and consumption. Therefore, the growing use of anxiolytics is worrisome, especially with regard to their irrational use, which can lead to tolerance, chemical dependency and withdrawal syndrome. This research aims to describe the uses of anxiolytic drugs sold in a Family Pharmacy in Southwest Bahia. This is a descriptive and cross-sectional study, using a quantitative approach. The data was collected through a questionnaire answered by the users of these drugs, in August and September 2021. The data analysis was performed using the Statistical Package for the Social Sciences version 22.0, and presented as absolute and relative frequency for the categorical variables, in addition to mean and standard deviation for the continuous variables. As a result, the study was able to identify the profile of the chronic user of benzodiazepines: a person with an average age of $53.87( \pm 11.10)$ years, with a predominance of females (59.34\%), with low purchasing power and low education level. The most used drug was Clonazepam and the most indicated pathology, insomnia. It is expected that the data obtained from this research could contribute to implement conducts and measures that promote its rational use and could also assist in the implementation of
\end{abstract}


treatment alternatives aimed at improving the quality of life of these patients, including the adoption of nonpharmacological actions.

Keywords: Anxiolytics; Use of medication study; Rational use.

\section{Resumen}

El uso racional de medicamentos debe ser promovido a través de iniciativas que regulen su prescripción, dispensa y consumo. Por ello, el uso creciente de ansiolíticos es preocupante, especialmente en lo que respecta a su uso irracional, que puede conducir a la tolerancia, dependencia química y síndrome de abstinencia. Esta investigación tiene como objetivo describir el tipo de uso que se hace con los ansiolíticos vendidos en una Farmacia Familiar en el Suroeste de Bahía. Se trata de un estudio descriptivo y transversal, con enfoque cuantitativo, cuyos datos fueron recogidos mediante un cuestionario respondido por los usuarios de estos fármacos, en agosto y septiembre de 2021 . El análisis de estos datos se realizó mediante el Paquete Estadístico para las Ciencias Sociales, versión 22.0, y se presentó en forma de frecuencia absoluta y relativa para las variables categóricas, además de la media y la desviación estándar para las variables continuas. Como resultado, se logró definir el perfil del usuario crónico de benzodiacepinas, con una edad promedio de 53,87 $( \pm 11,10)$ años, con predominio del sexo femenino $(59,34 \%)$, con bajo poder adquisitivo y bajo nivel educativo. El fármaco más utilizado fue el Clonazepam y la patología más indicada, el insomnio. Se espera que los datos obtenidos de esta investigación puedan contribuir a establecer conductas y medidas que promuevan su uso racional y ayuden a implementar alternativas de tratamientos dirigidas a mejorar la calidad de vida de estos pacientes, incluida la adopción de medidas no farmacológicas.

Palabras clave: Ansiolíticos; Estudio del uso de medicamentos; Uso racional.

\section{Introdução}

O Uso Racional de Medicamentos (URM) acontece quando seu consumo é adequado à situação clínica do paciente. Para que isso ocorra, a prescrição do medicamento deve ser realizada na dose e tempo de duração indicados ao tratamento e às necessidades individuais de cada usuário. Além disso, é preciso que a terapia medicamentosa seja acessível e ofertada a um menor custo possível (OMS, 1985).

O URM foi recomendado pela Organização Mundial de Saúde (OMS) para as políticas de medicamentos. No Brasil, essa orientação se encontra entre os objetivos e as diretrizes da Política Nacional de Medicamentos (PNM), bem como da Política Nacional de Assistência Farmacêutica (PNAF), e deve ser promovida por meio de ações que regulem sua prescrição, dispensação e consumo (Brasil, 1998a; Brasil, 2004; OMS, 1985).

Restringir-se exclusivamente ao diagnóstico e tratamento de doenças nem sempre é o bastante para garantir a efetividade no cuidado em saúde. Para a promoção do URM é imprescindível que exista uma interação direta entre o usuário do medicamento e o farmacêutico, profissional habilitado para a prática da atenção farmacêutica (Brasil, 2004). Ele é responsável por detectar e realizar as necessidades dos pacientes relacionadas à terapia medicamentosa, a fim de torná-la a mais indicada, efetiva, segura e conveniente, propiciando a melhora clínica (Cipolle et al., 2004; Correia et al., 2017).

Diante dessa premissa, é preocupante o crescente uso de medicamentos, em especial os ansiolíticos, principalmente no que diz respeito ao seu uso irracional, o qual pode acarretar tolerância, dependência química e síndrome de abstinência. Entre esses fármacos, os benzodiazepínicos (BZDs) são os mais consumidos por mulheres, com uma propensão para o crescimento da utilização no envelhecimento. Sendo assim, é necessária uma atenção maior e avaliações constantes durante o tratamento com esses medicamentos, já que eles ainda podem ocasionar déficit cognitivo e riscos de queda (Fiorelli \& Assini, 2017).

O último panorama publicado sobre os dados do Sistema Nacional de Gerenciamento de Produtos Controlados (SNGPC), realizado entre 2007 a 2010, informa que os BZDs mais dispensados no país nesse período (exceto as formulações manipuladas) foram o Clonazepam, seguido do Bromazepam e do Alprazolam (Brasil, 2011).

Os BZDs são indicados, com maior relevância, para o tratamento da insônia e ansiedade, sendo os mais prescritos da classe o Clonazepam e o Diazepam. Isso se deve à distribuição gratuita na rede pública, já que constam na Relação Nacional de Medicamentos Essenciais (RENAME), instrumento utilizado para nortear Estado e municípios na elaboração de suas listas de medicamentos, de acordo com sua situação epidemiológica (Brasil, 2020; Menezes, 2019). 
Apesar do papel relevante que os BZDs exercem na vida dos usuários desses fármacos, a forma como são utilizados chama atenção, sobretudo, para o seu uso crônico, além do rotineiro, concomitante a outros medicamentos (polifarmácia), o que pode ocasionar riscos de interações medicamentosas e efeitos adversos (Medeiros et al., 2018).

Os BZDs estão elencados no Regulamento Técnico de substâncias psicotrópicas sujeitas ao controle especial, norma que deve ser consultada por todos os profissionais de saúde, pois determina a maneira como devem ser prescritos e dispensados. Essas substâncias encontram-se discriminadas na Lista B1, sujeitas à notificação de receituário azul "B” em virtude dos riscos que podem gerar à saúde psicológica e física do usuário, principalmente quando seu uso é prolongado (Brasil, 1998b).

Diante da relevância desses dados, visto que estudos sobre a representação do uso desses fármacos na Bahia são insuficientes, principalmente na região Sudoeste, esta pesquisa apresenta como objetivo descrever o perfil de utilização dos medicamentos ansiolíticos dispensados em uma Farmácia da Família no Sudoeste Baiano.

\section{Metodologia}

Trata-se de um estudo descritivo e transversal, com abordagem quantitativa, que avaliou a utilização de ansiolíticos da classe dos BZDs por usuários atendidos em uma unidade da Farmácia da Família no Sudoeste Baiano, nos meses de agosto e setembro de 2021.

Segundo Gil (2008), o estudo descritivo reúne um conjunto de variáveis durante um período de tempo estabelecido e tem por finalidade a caracterização de uma determinada população ou fenômeno, ou ainda o estabelecimento de relações entre as variáveis. Para isso, utiliza-se a observação sistemática e técnicas uniformes na coleta das informações, como questionários. Nesse tipo de pesquisa, há apenas as observações, registros, análises e organização dos dados, sem interferência nas fontes.

O estudo transversal, conforme Bastos e Duquia (2007), envolve a população de interesse, a fim de determinar a prevalência de exposição e desfecho para cada uma das pessoas estudadas em um único momento no tempo, permitindo, assim, detectar grupos de risco e coletar informações que propiciem ações de planejamento em saúde.

A abordagem de natureza quantitativa considera, para Prodanov e Freitas (2013), a quantificação dos dados, traduzidos em números, opiniões e informações, além de exigir a aplicação de recursos e técnicas estatísticas.

A amostra foi composta por pacientes com prescrições de BZDs que buscaram a dispensação desses medicamentos nessa farmácia pública. Como critérios de inclusão estabeleceu-se idade superior a 18 anos e confirmação de participação mediante a assinatura do Termo de Consentimento Livre e Esclarecido (TCLE). Por outro lado, foram excluídos da pesquisa menores de 18 anos, pessoas legalmente incapazes e aqueles que não tiveram interesse em participar.

Os dados utilizados neste estudo foram extraídos por meio de entrevista aos usuários, aplicando-se um questionário dividido em três partes envolvendo perguntas abertas e fechadas. A primeira parte foi constituída por variáveis sociodemográficas, a segunda por informações sobre as condições de saúde do paciente e a utilização dos serviços médicos, já a terceira, por particularidades relacionadas ao uso dos BZDs prescritos aos participantes da pesquisa.

As informações obtidas foram avaliadas através do pacote estatístico Statistical Package for the Social Sciences (SPSS) versão 22.0. Executou-se a análise descritiva apresentada em forma de frequência absoluta e relativa para as variáveis categóricas, além da média e desvio padrão para as variáveis contínuas.

Os dados foram coletados após aprovação do Comitê de Ética em Pesquisa da Faculdade Independente do Nordeste (FAINOR), conforme parecer $\mathrm{n}^{\mathrm{o}}$ 4.899.574. Os procedimentos foram realizados em concordância com o preconizado na Resolução $n^{\circ}$ 466/2012 e 510/2010 do Conselho Nacional de Saúde. A fim de assegurar a integridade desse estudo, assim como esclarecer todas as informações inerentes à pesquisa, os participantes receberam o TCLE, elaborado em duas vias, as quais foram assinadas pelo entrevistado e pelo pesquisador responsável (Brasil, 2013b, 2016). 


\section{Resultados e Discussão}

Participaram dessa pesquisa, ao todo, 91 usuários de ansiolíticos da classe dos BZDs, com idade mínima de 24 e máxima de 83 anos, apresentando uma média de 53,26 ( $\pm 12,01)$ anos. Obteve-se como resultado uma prevalência de uso crônico de BZDs por parte do gênero feminino, na faixa etária entre 24-59 anos (59,02\%), católicas ou evangélicas, com baixos poder aquisitivo e grau de escolaridade. A maioria delas $(61,11 \%)$ informou não conviver com o companheiro (solteiras, separadas, divorciadas e viúvas), entretanto residem com outros familiares (duas a cinco pessoas por domicílio), conforme dados da Tabela 1.

Tabela 1 - Distribuição dos usuários de benzodiazepínicos entrevistados por variáveis sociodemográficas. Farmácia da Família, Região Sudoeste - BA, 2021.

\begin{tabular}{|c|c|c|c|}
\hline \multirow{3}{*}{$\begin{array}{l}\text { Variáveis } \\
\text { Demográficas }\end{array}$} & \multicolumn{3}{|c|}{ n (\%) } \\
\hline & \multirow{2}{*}{ Total } & \multicolumn{2}{|c|}{ Gênero } \\
\hline & & Feminino & Masculino \\
\hline \multicolumn{4}{|l|}{ Faixa Etária } \\
\hline 24 a 59 anos & $61(67,03)$ & $36(59,02)$ & $25(40,98)$ \\
\hline 60 a 83 anos & $30(32,97)$ & $18(60,00)$ & $12(40,00)$ \\
\hline \multicolumn{4}{|l|}{ Religião } \\
\hline Católica & $47(51,65)$ & $26(48,15)$ & $21(56,76)$ \\
\hline Evangélica & $34(37,36)$ & $22(40,74)$ & $10(27,02)$ \\
\hline Outros & $10(10,99)$ & $06(11,11)$ & $06(16,22)$ \\
\hline \multicolumn{4}{|l|}{ Estado Civil } \\
\hline Solteiro (a) & $29(31,87)$ & $14(25,93)$ & $15(40,54)$ \\
\hline Casado (a) / coabitado (a) & $36(39,56)$ & $20(37,03)$ & $16(43,24)$ \\
\hline Divorciado (a) /separado (a) & $20(21,98)$ & $15(27,78)$ & $05(13,51)$ \\
\hline Viúvo (a) & $05(05,49)$ & $04(07,41)$ & $01(02,70)$ \\
\hline Outros & $01(01,10)$ & $01(01,85)$ & $00(00,00)$ \\
\hline \multicolumn{4}{|l|}{ Socioeconômicas } \\
\hline \multicolumn{4}{|l|}{ Renda (em salários mínimos)* } \\
\hline 1 a 3 & $61(67,03)$ & $34(62,96)$ & $27(72,97)$ \\
\hline$<1$ ou não possui renda & $30(32,97)$ & $20(37,04)$ & $10(27,03)$ \\
\hline \multicolumn{4}{|l|}{ Grau de instrução } \\
\hline Nunca frequentou escola & $03(03,30)$ & $02(03,70)$ & $01(02,70)$ \\
\hline Ensino fundamental incompleto & $37(40,66)$ & $25(46,30)$ & $12(32,43)$ \\
\hline Ensino fundamental completo & $22(24,17)$ & $16(29,63)$ & $09(24,32)$ \\
\hline Ensino médio incompleto & $03(03,30)$ & & \\
\hline Ensino médio completo & $22(24,17)$ & $09(16,67)$ & $13(35,14)$ \\
\hline Ensino superior incompleto & $01(01,10)$ & $01(01,85)$ & $01(02,70)$ \\
\hline Ensino superior completo & $03(03,30)$ & $01(01,85)$ & $01(02,70)$ \\
\hline \multicolumn{4}{|l|}{ Reside } \\
\hline Sozinho (a) & $20(21,98)$ & $13(24,07)$ & $07(18,92)$ \\
\hline Com a família & $67(73,62)$ & $38(70,37)$ & $29(78,38)$ \\
\hline Outros & $04(04,40)$ & $03(05,56)$ & $01(02,70)$ \\
\hline Total & $91(100,00)$ & $54(100,00)$ & $37(100,00)$ \\
\hline
\end{tabular}

Em revisão sistemática sobre os aspectos clínicos dos BZDs, entre 2015 a 2019, Menezes (2019) aponta que o consumo desses medicamentos no Brasil é mais elevado entre as mulheres idosas, na faixa de 53 a 103 anos de idade, o equivalente a aproximadamente 76,2\%. Resultado semelhante foi encontrado por Morceli et al. (2020), cujo percentual de utilização por parte do gênero feminino foi de 82,3\%, enquanto que no levantamento realizado por Mendonça et al. (2020), esse correspondeu a $62 \%$. Esses achados corroboram com o atual estudo, embora os percentuais não sejam os mesmos, visto 
que o total de entrevistadas correspondeu a 59,34\%, ao passo que 40,66\% dos participantes eram do sexo masculino.

Sob o mesmo ponto de vista, Fiorelli e Assini (2017) complementam que há uma propensão para o crescimento da utilização desses fármacos diretamente relacionada com o envelhecimento, o que coincide com o perfil dessa pesquisa, pois entre as mulheres a idade média foi de 53,87 ( \pm 11,10). Além disso, estudos em outros países, como os de Alpízar et al. (2020) e Singh e Oosthuizen (2019), coincidem com esses dados.

Vale ressaltar que essa prevalência se enquadra no grupo etário de adultos no final da fase produtiva, situação que pode estar relacionada ao fenômeno da medicalização cotidiana, que engloba uma variedade de condições, condutas e experiências, em outros tempos, vistas como normais, mas que hoje são tratadas como patologias (Alpízar et al., 2020).

Por outro lado, esse desfecho mais elevado entre as mulheres também pode corresponder ao fato delas serem as maiores usuárias dos serviços de saúde, já que têm um melhor cuidado com seu bem-estar. Ao mesmo tempo, outras condições sociais, econômicas e culturais condicionam o padrão de saúde desse gênero, acometido mais constantemente por quadros de ansiedade e depressão (Fávero et al., 2018).

Considerando o perfil financeiro dos participantes, a maioria declarou receber entre um e três salários mínimos por mês, o correspondente a $67,03 \%$ em contraposição dos $32,91 \%$, cujos rendimentos são inferiores ou alegaram não possuir renda. Já sobre o grau de escolaridade, ficou comprovado que 68,13\% dos usuários não tiveram acesso ao ensino médio (sem instrução e ensino fundamental completo ou incompleto) e só 3,30\% ingressaram no ensino superior.

Da mesma forma, essa relação entre baixo grau de escolaridade e condição financeira com o consumo de BZDs é evidenciada em outros estudos. De acordo com Mendonça et al. (2019), a respeito dos rendimentos financeiros, 55,1\% dos participantes da pesquisa recebiam até um salário mínimo. Já para Morceli et al. (2020), o equivalente a 67,6\% dos entrevistados informou receber até três salários mínimos. Além do mais, ambos concluíram que grande parte dos pacientes não teve acesso ao ensino médio, correspondendo a um percentual de $68 \%$ e $49,9 \%$ para cada um dos autores, respectivamente.

Nesse sentido, a baixa escolaridade provoca interferências no processo saúde-doença, uma vez que, no âmbito da saúde, a linguagem utilizada é técnica e envolve expressões de difícil entendimento. Sendo assim, esse pode ser um fator que influencia a interpretação de informações, muitas vezes complexas, acerca do tratamento farmacológico (Mosfiak et al., 2020).

Com relação às características relacionadas aos serviços de saúde, 95,60\% dos participantes adquiriram a prescrição de benzodiazepínico após atendimento médico pelo Sistema Único de Saúde (SUS). A maioria declarou que a frequência das consultas era bimestral, correspondendo a $59,34 \%$ dos entrevistados.

Por meio da análise das notificações de receituário azul "B”, pode-se perceber um predomínio de prescrições por parte dos psiquiatras, representando 57,14\% das receitas, acompanhadas de 18,68\% de indicações por clínicos gerais, 14,29\% por médicos das estratégias da saúde da família (ESF) e 9,89 por outras especialidades. Esses achados divergem dos encontrados por Fávero et al. (2018), pois grande parte das prescrições, 47\%, foi efetuada por clínico geral, 25\% por psiquiatras, 15,6\% por neurologistas e $12,4 \%$ por outras especialidades.

Embora os resultados do presente estudo indiquem prevalência do prescritor psiquiatra, nota-se que outros profissionais também efetuaram a indicação de BZDs. Esse fato, associado à frequência das consultas bimestral, pode indicar a existência de apenas renovações de prescrições sem o devido acompanhamento desses pacientes pelo especialista. A procura acentuada desses fármacos, somada ao desconhecimento acerca dos riscos do seu consumo e às prescrições inadequadas de médicos não especialistas, colabora para o crescente uso indiscriminado de ansiolíticos, o qual aumenta as chances de reações adversas, intoxicações, bem como dependência (Moreira \& Borja, 2018). A Tabela 2 especifica como a prescrição dos BZDs foi adquirida pelos pacientes conforme o atendimento médico, bem como a especialidade do prescritor e a frequência das consultas. 
Tabela 2. Distribuição dos usuários de benzodiazepínicos entrevistados por serviço de saúde (atendimento, especialidade médica e frequência das consultas). Farmácia da Família, Região Sudoeste - BA, 2021.

\begin{tabular}{lc}
\hline Características & $\mathbf{n}(\%)$ \\
\hline Atendimento & $87(95,60)$ \\
$\quad$ SUS & $04(04,40)$ \\
$\quad$ Particular & \\
Especialidade do médico & $52(57,14)$ \\
$\quad$ Psiquiatra & $17(18,68)$ \\
Clínico Geral & $13(14,29)$ \\
Médico do ESF & $09(09,89)$ \\
Outros & \\
Frequência das consultas & $03(03,30)$ \\
Mensal & $54(59,34)$ \\
Bimestral & $17(18,68)$ \\
Trimestral & $03(03,30)$ \\
Quadrimestral & $06(06,59)$ \\
Semestral & $05(05,49)$ \\
Anual & $03(03,30)$ \\
Inicio de tratamento & $\mathbf{9 1}(\mathbf{1 0 0 , 0 0})$ \\
\hline Total &
\end{tabular}

Fonte: Autores (2021).

O estudo sobre o uso de medicamentos é uma preocupação global, visto que inúmeros fatores, como a dinâmica populacional, as questões sociais, econômicas, culturais e as políticas públicas de saúde influenciam no perfil de utilização. Segundo a última Pesquisa Nacional sobre Acesso, Utilização e Promoção do Uso Racional de Medicamentos no Brasil PNAUM, o consumo de medicamentos vem se elevando entre os brasileiros (Bermudez \& Barros, 2016).

Tal elevação do consumo está relacionada às mudanças geradas no comportamento da sociedade pela urbanização e o avanço da tecnologia, responsáveis por tornar a preocupação, a ansiedade, o estresse e suas consequentes alterações no sono, queixas generalizadas da vida moderna. Por conseguinte, a busca pelo alívio dessas sensações levou ao aumento da procura de medicamentos que minimizem os sintomas, dentre os quais os ansiolíticos se incluem (Nunes \& Bastos, 2016).

Os dados referentes à caracterização das condições de saúde demonstraram que quase a totalidade dos usuários de BZDs dessa pesquisa, 92,30\%, informou apresentar mais de um problema de saúde atualmente, e 7,69\% dos pacientes relataram somente uma patologia.

No que se refere ao problema de saúde mais citado pelos entrevistados, a insônia correspondeu a um percentual de 96,91\% do total de usuários de BZDs. A Figura 1 apresenta um gráfico de incidência de doenças conforme as informações obtidas durante a pesquisa. Confrontando esses dados com os da Tabela 3, esse é o motivo pelo qual quase a totalidade utiliza o medicamento.

Percebe-se também através da Figura 1, que a maioria $(97,67 \%)$ apresenta outros transtornos mentais, que podem levar ao quadro clínico de insônia. Nesse sentido, é fundamental a avaliação da situação de saúde do paciente por um especialista, com o propósito de distinguir se a insônia se apresenta como fator principal de uma síndrome ou secundário à presença de outras patologias. 
Figura 1. Morbidades por usuários de benzodiazepínicos entrevistados. Farmácia da Família, Região Sudoeste - BA, 2021.

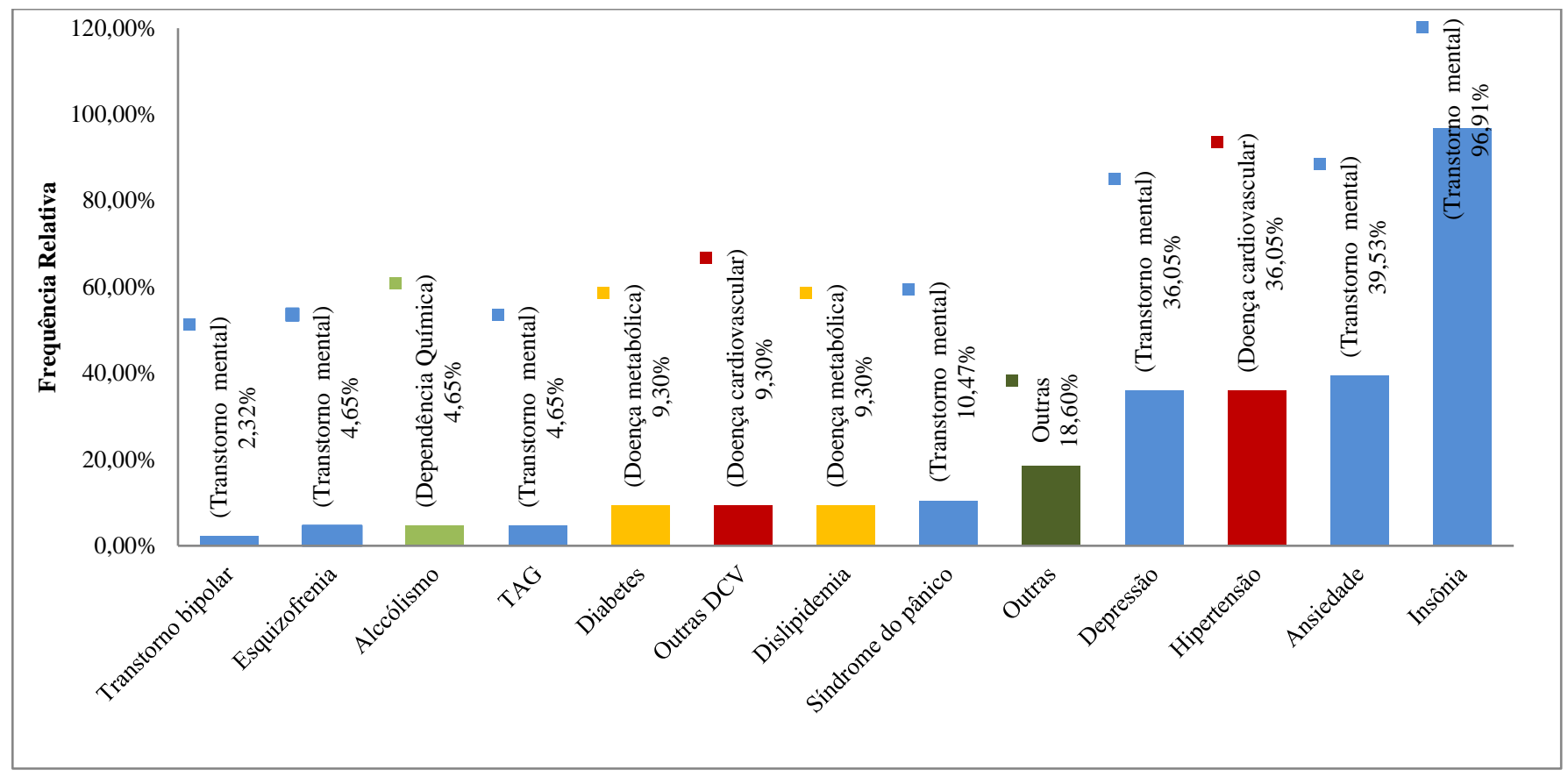

Fonte: Autores (2021).

Outros autores apontam resultados semelhantes aos encontrados nesse estudo. Medeiros et al. (2018) afirmam que a maioria dos entrevistados apresentou uma doença crônica, sendo a hipertensão arterial a patologia com maior percentual (35,5\%). Já Mosfiak et al. (2020) identificaram em sua pesquisa que 85,49\% dos participantes tinham algum problema de saúde atualmente, com destaque para a depressão (39,62\%), a hipertensão $(35,84 \%)$ e os problemas de coluna (16,98\%).

Ainda sobre as condições de saúde dos entrevistados, é importante alertar para o consumo de BZDs com outros fármacos. Entre os participantes do estudo, 87,91\% afirmaram fazer o uso de outros medicamentos, sendo que $31,87 \%$ deles relataram utilizar cinco ou mais fármacos (incluindo o benzodiazepínico), caracterizando a polifarmácia. Entre os mais utilizados, os antidepressivos corresponderam a um percentual de 42,86\%, seguido pelos hipertensivos (32,97\%), antipsicóticos $(27,47 \%)$ e anticonvulsivante $(19,78 \%)$.

Para Morceli et al. (2020) os BZDs são fármacos com propriedades farmacocinéticas e farmacodinâmicas favoráveis a interação medicamentosa, logo os riscos devem ser avaliados quando seu uso for associado a outros medicamentos pertencentes às classes dos anticonvulsivantes, antidepressivos, anticoagulantes orais, antiulcerogênicos e anti-inflamatórios.

É significativo o crescente consumo de medicamentos entre os brasileiros: mais da metade da população usa ao menos um medicamento. Ressalta-se também, o progressivo envelhecimento populacional, que requer atenção especial, pois o grupo de indivíduos que mais os utiliza, incluindo a polifarmácia, são os idosos. Além disso, dentre eles, aproximadamente $20 \%$ consomem pelo menos cinco fármacos. Inquéritos de saúde envolvendo avaliação sobre medicamentos são ferramentas imprescindíveis para coleta de dados e informações a respeito do seu uso, fundamentais para nortear os programas públicos de saúde e o URM (Bermudez \& Barros, 2016).

Acerca das particularidades relacionadas ao uso de BZDs, o Clonazepam foi o medicamento mais prescrito para os pacientes deste estudo, representando $85,71 \%$ das notificações de receitas B1, seguido por 17,58\% de indicações do Diazepam. Entretanto, não ocorreu a dispensação de 16,49\% dessas prescrições por motivos diversos, como rasuras, ausência do carimbo do prescritor, concentração errada do fármaco, dosagem não pertencente à Relação Municipal de Medicamentos Essenciais (REMUME), Cartão Nacional de Saúde (CNS) não pertencente ao município e nova prescrição antes do prazo previsto para o término da última medicação dispensada (menos de 60 dias). Os pacientes foram orientados quanto às irregularidades para que 
pudessem providenciar as devidas correções. A Tabela 3 demonstra o perfil de prescrição e dispensação dos BZDs por usuários participantes da pesquisa.

Tabela 3. Distribuição das prescrições por benzodiazepínicos dispensados / não dispensados, tempo de utilização e motivo de uso desses medicamentos por usuários entrevistados. Farmácia da Família, Região Sudoeste - BA, 2021.

\begin{tabular}{|c|c|c|c|}
\hline \multirow{2}{*}{ Características } & \multicolumn{3}{|c|}{ n (\%) } \\
\hline & Prescrição & Dispensado & Não dispensado \\
\hline \multicolumn{4}{|l|}{ Benzodiazepínicos } \\
\hline Clonazepam & $78(85.71)$ & & \\
\hline $2 \mathrm{mg}$ & & $37(48,35)$ & $07(07,69)$ \\
\hline $2,5 \mathrm{mg} / \mathrm{mL}$ & & $26(28,57)$ & $04(04,40)$ \\
\hline $0,5 \mathrm{mg}$ & & & $04(04,40)$ \\
\hline Diazepam & $16(17,58)$ & & \\
\hline $5 \mathrm{mg}$ & & $02(02,20)$ & \\
\hline $10 \mathrm{mg}$ & & $14(15,38)$ & \\
\hline Tempo de utilização & & Motivo de uso & \\
\hline Continuo & $82(90,11)$ & Ansiedade & $50(54,94)$ \\
\hline Intermitente & $07(07,69)$ & Depressão & $24(26,37)$ \\
\hline \multirow[t]{2}{*}{ Início de tratamento } & $02(02,20)$ & Insônia & $83(91,21)$ \\
\hline & & Outros & $06(06,59)$ \\
\hline Total & $91(100,00)$ & & \\
\hline
\end{tabular}

Fonte: Autores (2021).

Estudos sobre a utilização desses medicamentos em outros países apresentam resultados equivalentes, com destaque para a indicação do Clonazepam, seguido pelo Diazepam e Lorazepam, na segunda e/ou terceira posições (Alpízar et al., 2020; Singh \& Oosthuizen, 2019).

Similarmente, os autores Alvim et al. (2017), Gonçalves (2019), Moreira e Borja (2018) e Schallemberger e Colet (2016) compartilham esses mesmos resultados, evidenciando que, no Brasil, o Clonazepam e o Diazepam estão nas primeiras posições do ranking, correlacionadas em vários estudos à sua disponibilização e oferta gratuita pelo sistema público de saúde, uma vez que estão elencados na RENAME. Evidencia-se, portanto, a prevalência (até mesmo mundial) do consumo desses fármacos de meia-vida longa, prescritos e dispensados a partir de uma favorecida condição do sistema de saúde.

Ainda vale ressaltar, que os BZDs diferenciam-se uns dos outros devido às suas propriedades farmacocinéticas (meiavida, tempo de absorção e via de eliminação) e à potência do fármaco. Essas propriedades, somadas à natureza da doença e ao julgamento médico, são fatores determinantes para a escolha e prescrição desses medicamentos a fim de obter sucesso no tratamento (Baes \& Juruena, 2017; Sadock et al., 2017).

O período que o fármaco permanece na corrente sanguínea até que metade dele seja eliminada (tempo de meia-vida plasmática) é um fator importante a ser considerado. Tanto o Diazepam e seus metabólitos, quanto o Clonazepam apresentam meia-vida longa, pois se ligam extensamente às proteínas plasmáticas (Lindner, 2017).

A meia-vida longa é uma vantagem, uma vez que o tratamento envolve doses menos frequentes, além de sintomas menos intensos de abstinência. Apesar disso, também gera uma maior concentração do medicamento no organismo, promovendo uma maior sedação e aumento dos riscos de comprometimento psicomotor (Baes \& Juruena, 2017).

O uso desses fármacos por pacientes idosos, apesar de frequente, requer um acompanhamento maior, visto que há riscos de quedas e fraturas, prejuízos cognitivos e dependência (Fiorelli \& Assini, 2017).

A atenção não só deve estar voltada para esses riscos, mas também para o de intoxicação, em virtude das alterações fisiológicas que ocorrem com o avançar da idade. A ação das enzimas microssomais hepáticas declina de forma lenta e variável. Ocorre também elevação do volume de distribuição desses fármacos lipossolúveis, devido a maior proporção de 
gordura corporal, não deixando de considerar o aumento da meia-vida dos BZDs. Dessa forma, a farmacodinâmica e farmacocinética dessas substâncias também sofrem variações. Logo, o Diazepam e Clonazepam não devem ser prescritos para os idosos, já que eles necessitam de metabolização hepática intensa (Nunes \& Bastos, 2016; Rang et al., 2016).

Por sua vez, ainda a partir da análise da Tabela 3, percebe-se que o tempo de utilização desses medicamentos excede o descrito na literatura. Isso porque, houve prevalência de uso contínuo entre $90,11 \%$ dos pacientes, os quais informaram o consumo por um período acima de um ano, enquanto que 7,69\% declaram uso intermitente e apenas $2,20 \%$ iniciaram o tratamento a pouco tempo do período de estudo.

Esses dados condizem com os encontrados por Mendonça et al. (2020) que apontam um perfil de uso crônico de BZDs, complementado que $36 \%$ dos entrevistados o faziam a mais de 10 anos, sendo que a maioria relatou não ser capaz de viver sem a medicação (74\%).

Mosfiak et al. (2020) também evidenciam essa cronicidade, no entanto, destacam que houve relatos de consumo por mais de 20 anos, o que gera como consequência a dependência.

Os BZDs podem apresentar efeitos indesejáveis mesmo quando utilizados em doses terapêuticas normais. Além disso, em caso de uso indevido ou por tempo prolongado, esses efeitos são potencializados, podendo acarretar dependência e como consequência, síndrome de descontinuação (Nunes \& Bastos, 2016).

Outras reações adversas ocasionadas pelo uso crônico de BZDs incluem: ansiedade rebote, náusea, declínio cognitivo, quedas, alterações de percepção e, eventualmente, convulsões epilépticas e psicose. Ainda podem ocorrer neoplasias malignas, comorbidade de humor e transtorno neurótico. (Menezes, 2019).

No que diz respeito ao motivo pelo qual o paciente usa o medicamento, a maioria, 91,21\%, declarou utilizar para insônia, 54,94\% para ansiedade e 26,37\% para depressão. Além disso, mais da metade dos entrevistados, 54,94\%, afirmaram utilizar o benzodiazepínico por mais de um motivo.

Esses resultados divergem dos encontrados por Mendonça et al. (2020), que apontam como principal motivo de indicação desses fármacos a ansiedade (36,7\%), seguida pela insônia (28,5\%) e pela síndrome do pânico (14,3\%). Já Fávero et al. (2018) destacam que a insônia representou um maior percentual de utilização (62,5\%), acompanhada por depressão (53,1\%) e ansiedade (43,8\%). Ainda por cima, esclarecem que nesse quesito os entrevistados poderiam responder mais de um motivo pelo qual utilizavam o medicamento.

Os psicofármacos BZDs se enquadram na classe dos ansiolíticos que atuam deprimindo o Sistema Nervoso Central (SNC), o qual passa a ter sua ação inibida ou reduzida. Portanto, ocorre a diminuição da concentração, atenção e atividade motora dos usuários, associada a alguma ação analgésica (Costa, 2017).

A sua principal indicação terapêutica é como ansiolítico, empregado em situações relacionadas ao estresse, crises psicóticas, ou condições de transtorno de ansiedade primária. Ainda são recomendados para insônia, devido à ação sedativa e relaxante, bem como para controlar estados convulsivos, inclusive aqueles decorrentes da síndrome de abstinência ao álcool, cocaína ou crack (Brasil, 2013a).

Considerando outros dados obtidos acerca das particularidades relacionadas ao uso de BZDs, conclui-se que a maioria $(63,74 \%)$ dos participantes informou alteração ou aumento de doses desses fármacos com orientação médica $(58,62 \%)$, em contraposição aos que o declararam ter realizado por conta própria $(37,93 \%)$ e ambas as situações $(3,45 \%)$. Já quanto à tentativa de parar de usar o medicamento, 60,44\% dos usuários afirmaram ter iniciado o desmame, contudo mais da metade $(52,73 \%)$ o fez por conta própria, seguido dos que tentaram por orientações médicas (40\%) e por ambas as situações $(7,27 \%)$.

Quanto às informações sobre o processo de desmame, quase a metade dos pacientes $(49,09 \%)$ relatou que a orientação médica foi para redução gradual do benzodiazepínico e outros $(3,64 \%)$ informaram que o método adotado foi a substituição do 
medicamento. Os demais entrevistados buscaram a interrupção do tratamento por alguns dias (por conta própria), atitude errônea e que leva ao retorno do tratamento farmacológico.

Percebe-se que a taxa de insucesso do desmame foi bastante elevada, quase a totalidade dos que tentaram $(89,09 \%)$, sendo que o principal motivo foi a insônia (94,87\%). Esse fato também pode estar associado ao uso crônico do medicamento, pois quanto maior for o tempo de utilização, mas se torna difícil a descontinuação do tratamento, já que existem riscos de crises de abstinência (Nunes \& Bastos, 2016). A Tabela 4 indica a caracterização desses dados.

Tabela 4. Distribuição dos usuários entrevistados por particularidades relacionadas ao uso do benzodiazepínico. Farmácia da Família, Região Sudoeste - BA, 2021.

\begin{tabular}{lc}
\hline Características & $\mathbf{n}(\mathbf{\%})$ \\
\hline Alteração ou aumento de doses & $\mathbf{5 8}(\mathbf{6 3 , 7 4 )}$ \\
\hline Orientação Médica & $34(58,62)$ \\
Conta própria & $22(37,93)$ \\
Ambos & $02(03,45)$ \\
\hline Tentativa de parar de usar o medicamento & $\mathbf{5 5}(\mathbf{6 0 , 4 4 )}$ \\
\hline Orientação Médica & $22(40,00)$ \\
Conta própria & $29(52,73)$ \\
Ambos & $04(07,27)$ \\
Orientação & \\
Redução gradual & $27(49,09)$ \\
Substituição por outro medicamento & $02(03,64)$ \\
Outros & $26(47,27)$ \\
Sucesso no processo de retirada & \\
Sim & $06(10,91)$ \\
Não & $49(89,09)$ \\
\hline Sintoma (s) se não utiliza o medicamento & $\mathbf{7 8}(\mathbf{8 5 , 7 1 )}$ \\
\hline Insônia & $74(94,87)$ \\
Ansiedade & $48(61,53)$ \\
Depressão & $09(11,54)$ \\
Agitação & $30(38,46)$ \\
Outros & $16(20,51)$ \\
\hline Sintoma (s) quando utiliza o medicamento & $\mathbf{3 7}(\mathbf{4 0 , 6 6 )}$ \\
\hline Perda de memória & $26(70,27)$ \\
Sonolência & $13(35,14)$ \\
Outros & $08(21,62)$ \\
\hline Orientações recebidas do médico & $50(54,94)$ \\
\hline Não dirigir, operar máquinas, etc. & $51(56,04)$ \\
Não consumir bebidas alcoólicas durante o tratamento & $57(62,64)$ \\
\hline Não usar por período prolongado este medicamento & $\mathbf{9 1 ( 1 0 0 , 0 0 )}$ \\
\hline Total & \\
\hline & \\
\hline
\end{tabular}

Fonte: Autores (2021).

Com relação ao espectro de sintomas descritos pelos entrevistados quando não fazem o uso do fármaco, além da insônia os mais comuns foram ansiedade (61,53\%), agitação $(38,46 \%)$ e depressão $(11,54 \%)$. Essa situação também se assemelha à encontrada por Medeiros et al. (2018), visto que, em seus estudos, $30 \%$ dos usuários tentaram interromper o uso do benzodiazepínico e não conseguiram em virtude do surgimento de sintomas, como: nervosismo, insônia, agitação, inquietude, pânico, cefaleia e tremores.

Estudo realizado por Kowalski et al. (2020) apontou que cerca de um terço (36\%) dos entrevistados foram orientados pelo prescritor ao desmame com redução gradual, porém a orientação não teve sucesso, pois os mesmo relataram cansaço, insônia, agitação, ansiedade, sintomas que levaram à interrupção do processo. Além do mais, não houve tentativa de redução gradual ou interrupção do tratamento com orientação médica para os demais casos (64\%), permanecendo a prescrição de uso 
contínuo. Ainda assim, 20,83\% dos pacientes tentaram a descontinuação medicamentosa por conta própria, preocupados com a dependência que o fármaco poderia ocasionar, não obtendo sucesso.

Além dos sintomas de abstinência já apresentados, outros também ocorrem com a interrupção da farmacoterapia, como: sudorese, palpitações, letargia, náuseas, irritabilidade e dificuldade de concentração. Essa sintomatologia evolui entre o quinto e o décimo dia de interrupção dos BZDs de meia-vida longa, como o Clonazepam e o Diazepam (Fiorelli \& Assini, 2017; Sadock et al., 2017).

A indicação do medicamento envolve o diagnóstico clínico relacionado aos sintomas-alvo e o tempo da farmacoterapia. Tudo deve ser discutido e decidido com a coparticipação do paciente. Normalmente, o tratamento para as condições de ansiedade dura em torno de 2 a 6 semanas e a conduta para a descontinuação do medicamento deve ser gradativa até a interrupção total, entre 1 a 2 semanas. É fundamental a vigilância quanto a prováveis sintomas de abstinência, além de oferecer assistência psicológica para o paciente, pois dessa forma o resultado da descontinuação do tratamento terá êxito. Persistir com a utilização de BZDs por um tempo prolongado é um engano, diante de tantos riscos que esses medicamentos podem ocasionar (Guina \& Merril, 2018; Sadock et al., 2017).

Outro fator importante entre os achados é a alteração ou aumento de doses desses fármacos, o que pode corresponder ao desenvolvimento de tolerância, reação adversa ao medicamento (RAM) que desperta preocupação. Relacionada a reações dependentes de dose e tempo, ela ocorre devido à administração repetida de BZDs, a qual diminui sua eficácia. Tal reação significa uma baixa resposta farmacológica, efeito de adaptação dinâmica dessas substâncias no organismo em razão do seu uso prolongado. Ocorrem alterações na estrutura do receptor Gabaérgico tipo A $\left(\mathrm{GABA}_{\mathrm{A}}\right)$, que sofre dessensibilização por causa da presença desses medicamentos. Esse processo pode estar associado ao desenvolvimento da dependência física e psicológica, pois há uma necessidade de prosseguir com sua utilização a fim de evitar o surgimento de sintomas de abstinência (Baes \& Juruena, 2017; Gonçalves, 2019).

Em relação aos seus efeitos hipnóticos, a tolerância pode evoluir com a utilização do medicamento no período de dias a semanas, enquanto que para os efeitos ansiolíticos ocorre de semanas a meses. Isso esclarece a elevação das doses desses fármacos pelo próprio indivíduo no decorrer do tratamento, mesmo sem orientação médica (Guina \& Merril, 2018).

O uso indiscriminado desses ansiolíticos é prejudicial à saúde do paciente em razão da sua capacidade de gerar tolerância e dependência em curto prazo. Mesmo assim, não há uma responsabilização por parte dos profissionais de saúde pelo seu desmame de maneira adequada, prevalecendo a prescrição por uso contínuo (Morceli et al., 2020).

A respeito dos sintomas apresentados pelos entrevistados quando utilizam o medicamento, menos da metade (40,66\%) informou apresentá-los. Os mais comuns foram perda de memória (70,27\%) e sonolência (35,14\%). Medeiros et al. (2018) também apontam reações adversas apresentadas pelos usuários de psicotrópicos, como sonolência $(9,4 \%)$, boca seca $(8,4 \%)$, ganho de peso $(4,4 \%)$ e tontura $3,9 \%$.

Sobre as orientações recebidas do médico, metade dos entrevistados $(54,94 \%)$ foi informada sobre os riscos de dirigir veículos ou operar máquinas, dos quais $92 \%$ foram advertidos no início do tratamento e $10 \%$ durante o tratamento. Além do mais, 56,04\% dos participantes do estudo foram alertados para não consumir bebidas alcoólicas durante o tratamento e 62,64\% para não utilizar o medicamento por um tempo prolongado.

Esses resultados são preocupantes, já que todos deveriam ter sido advertidos sobre os efeitos colaterais e riscos que esses fármacos podem ocasionar, pois se sabe que a sedação, sonolência, perda de memória, ataxia, diminuição da atenção e da concentração se encontram entre os principais efeitos colaterais gerados pelos BZDs (Baes \& Juruena, 2017).

Outrossim, é importante alertar sobre os efeitos potencializadores desses medicamentos quando administrados concomitantemente com o etanol ou até outras substâncias, visto que podem gerar uma interação grave e até mesmo letal, como a depressão respiratória (Kowalski et al., 2020). 
Promover o uso racional de medicamentos, antes de tudo, é avaliar todas as possibilidades sobre os benefícios farmacológicos, assim como sobre os riscos que os BZDs podem gerar à saúde do paciente ao prescrever esses fármacos, não suprimindo seu potencial de abuso e dependência (Guina \& Merril, 2018).

Nesses termos é notória a atenção a ser dada à prescrição e dispensação dos BZDs por parte dos profissionais de saúde, sobretudo, os médicos e farmacêuticos. Eles devem alertar sobre os prejuízos que esses compostos podem ocasionar, principalmente quando utilizados de forma inadequada, promovendo dessa forma, o seu uso racional (Nunes \& Bastos, 2016).

\section{Conclusão}

Com esse estudo foi possível traçar o perfil dos usuários de BZDs em uma unidade da Farmácia da Família no Sudoeste Baiano, observando-se um uso prolongado desta classe de medicamentos que possuem, dentre as principais características, o potencial de causar dependência. Ademais, ficou evidente a dificuldade quanto ao desmame desses medicamentos, mesmo com orientação médica, bem como a carência de informações sobre os riscos que podem gerar à saúde da população.

Sendo assim, espera-se que os resultados desta pesquisa possam contribuir com condutas e medidas que propiciem o seu uso racional e auxiliem na efetivação de alternativas de tratamento que visem à melhoria da qualidade de vida desses pacientes, inclusive com a adoção de medidas não farmacológicas. Contudo, outros estudos devem ser feitos com a finalidade de avaliar a qualidade do acompanhamento clínico contínuo dos usuários de BZDs, bem como de realizar ponderações sobre a manutenção prolongada de seu uso, além de identificar perspectivas de descontinuação da terapia medicamentosa.

\section{Referências}

Alvim, M. M., Cruz, D. T. da, Vieira, M. de T., Bastos, R. R., \& Leite, I. C. G. (2017). Prevalência e fatores associados ao uso de benzodiazepínicos em idosos da comunidade. Revista Brasileira de Geriatria e Gerontologia, 20(4), 463- 473. https://doi.org/10.1590/1981-22562017020.170042.

Alpízar, C. C., Valenciano, L. R., Fernández, M. C., \& Soto, L. E. H. (2020). Prescripciones de benzodiacepinas y barbitúricos en Costa Rica 20112015. Horizonte sanitario, 19(2), 277-290. https://doi.org/10.19136/hs.a19n2.3685.

Baes, C. V. W. \& Juruena, M. F. P. (2017). Psicofarmacoterapia para o clínico geral. Medicina (Ribeirão Preto. Online), 50, 22-36. https://doi.org/10.11606/issn.2176-7262.v50isupl1.p22-36.

Bastos, J. L. D. \& Duquia, R. P. (2007). Um dos delineamentos mais empregados em epidemiologia: estudo transversal. Scientia Medica, 17(4), 229-232. file://C:/Users/ASUS/Downloads/2806-Article\%20Text-42422-2-10-20130619\%20(1).pdf.

Bermudez, J. A. Z \& Barros, M. B. de A. (2016). Perfil do acesso e da utilização de medicamentos da população brasileira - contribuições e desafios da PNAUM - Inquérito Domiciliar. Rev. Saúde Pública, 50(supl 2):2s. https://doi.org/10.1590/s1518-8787.201605000supl2ap.

Brasil. (2011). Ministério da Saúde. Agência Nacional de Vigilância Sanitária. Boletim de Farmacoepidemiologia do Sistema Nacional de Gerenciamento de Produtos Controlados, Vol. 2, ano 1.

Brasil. (2004). Ministério da Saúde. Conselho Nacional de Saúde. Resolução no 338, de 06 de maio de 2004. Aprova a Política Nacional de Assistência Farmacêutica. Diário Oficial da União, Seção 1, p.52.

Brasil. (2013a). Ministério da Saúde. Conselho Nacional de Saúde. Resolução $n^{o} 466$, de 12 de dezembro de 2012. Dispõe sobre as diretrizes e as normas regulamentadoras de pesquisa envolvendo seres humanos. Diário Oficial da União, Seção 1, p.59.

Brasil. (2016). Ministério da Saúde. Conselho Nacional de Saúde. Resolução no 510, de 07 de abril de 2016. Ética na Pesquisa na área de Ciências Humanas e Sociais. Diário Oficial da União, Seção 1, p. 44, 45, 46.

Brasil. (1998a). Ministério da Saúde. Portaria GM/MS nº 3.916, de 30 de outubro de 1998. Aprova a Política Nacional de Medicamentos. Diário Oficial da União, Seção 1, p.18.

Brasil. (2013b). Ministério da Saúde. Secretaria de Atenção à Saúde. Departamento de Atenção Básica. Saúde mental. Departamento de Ações Programáticas Estratégicas. 176 p.: il. (Cadernos de Atenção Básica, n. 34).

Brasil. (2020). Ministério da Saúde. Secretaria de Ciência, Tecnologia, Inovação e Insumos Estratégicos em Saúde. Departamento de Assistência Farmacêutica e Insumos Estratégicos. Relação Nacional de Medicamentos Essenciais: Rename 2020 [recurso eletrônico]. Ministério da Saúde, 2017p. https://bvsms.saude.gov.br/bvs/publicacoes/relacao_medicamentos_rename_2020.pdf

Brasil. (1998b). Ministério da Saúde. Secretaria de Vigilância Sanitária. Portaria $n^{\circ} 344$ de 12 de maio de 1998. Aprova o Regulamento Técnico sobre substâncias e medicamentos sujeitos a controle especial. Diário Oficial da União, n. 91, Seção 1, p. 3. 
Cipolle, R. J., Strand, L. M. \& Morley, P. C. (2006). O Exercício do Cuidado Farmacêutico. - Tradução: Denise Borges Bittar; Revisão Técnica: Arnaldo Zubioli - Conselho Federal de Farmácia.

Correia, K. K. de L., Barros, M. L. C. M. G. R., Barros, M. R., Jr \& Marques, R. A.(2017). Farmácia clínica: importância deste serviço no cuidado a saúde. Boletim Informativo Geum, 8(3), 7-18. https://revistas.ufpi.br/index.php/geum/article/view/6183/4256.

Costa, J. M. (2017). Psicofarmacologia. Editora e Distribuidora Educacional S.A., 160p. ISBN 978-85-8482-857-9. http://cm-klscontent.s3.amazonaws.com/201701/INTERATIVAS_2_0/PSICOFARMACOLOGIA/U1/LIVRO_UNICO.pdf.

Fávero, V., Sato, M., \& Santiago, R. (2018). Uso de ansiolíticos: abuso ou necessidade? Visão Acadêmica, 18(4). http://dx.doi.org/10.5380/acd.v18i4.57820.

Fiorelli, K. \& Assini, F. (2017). A prescrição de benzodiazepínicos no Brasil: uma análise da literatura. ABCS Health Sci, 42(1),4044. http://dx.doi.org/10.7322/abcshs.v42i1.948.

Gil, A. C. (2008). Métodos e técnicas de pesquisa social. (6a ed.). Atlas. https://ayanrafael.files.wordpress.com/2011/08/gil-a-c-mc3a9todos-e-tc3a9cnicas-depesquisa-social.pdf.

Gonçalves, J. G. (2019). Benzodiazepínicos: malefícios relacionados à prática da automedicação e à falta de orientação adequada em saúde. Monografia (Graduação em Farmácia), Universidade Federal de Ouro Preto. http://www.monografias.ufop.br/handle/35400000/1891.

Guina, J., \& Merrill, B. (2018). Benzodiazepines II: Waking Up on Sedatives: Providing Optimal Care When Inheriting Benzodiazepine Prescriptions in Transfer Patients. Journal of clinical medicine, 7(2), 20. https://doi.org/10.3390/jcm7020020.

Kowalski, L., Schneider, M. S. \& Alves, I. A. (2020). Perfil dos usuários de benzodiazepínicos que frequentam uma drogaria da região Noroeste do Rio Grande do Sul. Ciência em Movimento - Reabilitação e Saúde, 22(43). https://doi.org/10.15602/1983-9480/cm.v22n43p149-160.

Lei $n^{o} 14.158$, de 2 de junho de 2021. Que define o valor do salário-mínimo em R\$1.100,00, a partir de $1^{\circ}$ de janeiro de 2021. DOU 4.6.2021, seção 1, ed. extra, pág. 1 .

Lindner, P. M. (2017). Benzodiazepínicos: uma revisão quanto aos aspectos farmacológicos, ao risco, dependência e abuso. Trabalho de Conclusão de Curso, Bacharelado em Farmácia, Faculdade de Educação e Meio Ambiente. http://repositorio.faema.edu.br:8000/jspui/handle/123456789/1243.

Medeiros, J. S. de A., Fo , Azevedo, D. M. de, Pinto, T. R. \& Silva, G. W. dos S. (2018). Uso de psicofármacos na atenção primária à saúde. Revista Brasileira em Promoção da Saúde, 31(3), 1-12. https://doi.org/10.5020/18061230.2018.7670.

Mendonça, P. N. T. de, Queiroz, L. J. V., Fo, Nascimento, A. M. dos S., Oliveira, T. M. de, \& Melo, D. S. B. de (2019). Características do uso de benzodiazepínicos por pacientes atendidos na unidade de saúde da família Djalma de Holanda Cavalcante em Recife-Pe. In book: Bases Conceituais da Saúde, 3(9), 72-82. https://doi.org/doi: 10.22533/at.ed.3431915029.

Menezes, C. S. (2019). Benzodiazepínicos: uma revisão sistemática (77p.). Trabalho de Conclusão de Curso, Bacharelado em Farmácia, Faculdade de Educação e Meio Ambiente. http://repositorio.faema.edu.br:8000/jspui/handle/123456789/2460.

Morceli, A. R., Ventorin, B. G., Ferreira, E. D. F. \& Silva, M. F. P. T. B. da (2020). Perfil socio-demográfico de usuários de benzodiazepínicos em uma unidade básica de saúde da cidade de Maringá, Pr. International Journal of Development Research, 10(10), 41092-41097. https://doi.org/10.37118/ijdr.20149.10.2020

Moreira, P. \& Borja, A. (2018). Benzodiazepínicos: uso e abuso em pacientes idosos. Revista Acadêmica Oswaldo Cruz, ano 5, n.19, ISSN 2357-8173 (versão on-line). http://revista.oswaldocruz.br/Content/pdf/Edicao_19_Pamella_Moreira.pdf.

Mosfiak, M. A., Brzozowski, F. S. \& Cichota, L. C. (2020). Análise do consumo de benzodiazepínicos em um município do norte do Rio Grande do Sul, Brasil. Rev. Saúde Col. UEFS, 10(1), 49-57. https://doi.org/10.13102/rscdauefs.v10i1.5214.

Nunes, B. S. \& Bastos, F. M. (2016). Efeitos colaterais atribuídos ao uso indevido e prolongado de benzodiazepínicos. Saúde e ciência em ação, 3(1), 71-82. ISSN: 2447 9330. http://www.revistas.unifan.edu.br/index.php/RevistaICS/article/viewFile/234/177.

Organización Mundial de La Salud - OMS. (1985). Uso racional de los medicamentos: informe de la Conferencia de Expertos, Nairobi, Ginebra: OMS. https://apps.who.int/iris/bitstream/handle/10665/37403/9243561057_spa.pdf?sequence=1\&isAllowed=y.

Prodanov, C. C. \& Freitas, E. C. de (2013). Metodologia do trabalho científico [recurso eletrônico]: métodos e técnicas da pesquisa e do trabalho acadêmico. ( $2^{\mathrm{a}}$ ed.). Feevale. https://aedmoodle.ufpa.br/pluginfile.php/291348/mod_resource/content/3/2.1-E-book-Metodologia-do-Trabalho-Cientifico-2.pdf.

Rang, H. P., Ritter, J. M., Flower, R. J. \& Henderson, G. (2016). Farmacologia. [Tradução Gea Consultoría Editorial]. (8a ed.). Elsevier. https://cssjd.org.br/imagens/editor/files/2019/Abril/Farmacologia.pdf.

Sadock, B. J., Sadock, V. A. \& Ruiz, P. (2017). Compêndio de psiquiatria: ciência do comportamento e psiquiatria clínica. [recurso eletrônico] / Tradução: Marcelo de Abreu Almeida et al. (11 a ed.). Artmed, https://oitavaturmadepsicofm.files.wordpress.com/2019/03/compecc82ndio-de-psiquiatria-kaplan-esadock-2017.pdf.

Schallemberger, J. B., Colet, C.de F. (2016). Assessment of dependence and anxiety among benzodiazepine users in a provincial municipality in Rio Grande do Sul, Brazil. Trends in Psychiatry and Psychotherapy, 38(02), 63-70. http://dx.doi.org/10.1590/2237-6089-2015-0041.

Singh, I., \& Oosthuizen, F. (2019). A retrospective review on benzodiazepine use: A case study from a chronic dispensary unit. South African medical journal, 109(2), 127-132. https://doi.org/doi:10.7196/SAMJ.2019.v109i2.13347 